\title{
Above-bandgap ordinary optical properties of GaSe single crystal
}

\author{
S. G. Choi, ${ }^{1, a)}$ D. H. Levi, ${ }^{1}$ C. Martinez-Tomas,${ }^{2}$ and V. Muñoz Sanjosé ${ }^{2}$ \\ ${ }^{1}$ National Center for Photovoltaics, National Renewable Energy Laboratory, Golden, Colorado 80007, USA \\ ${ }^{2}$ Departamento Física Aplicada y Electromagnetismo, Universitat de València, c/Dr. Moliner No. 50, \\ 46100 Burjassot, Spain
}

(Received 22 July 2009; accepted 29 July 2009; published online 10 September 2009)

\begin{abstract}
We report above-bandgap ordinary optical properties of $\varepsilon$-phase GaSe single crystal. Reference-quality pseudodielectric function $\langle\varepsilon(E)\rangle=\left\langle\varepsilon_{1}(E)\right\rangle+i\left\langle\varepsilon_{2}(E)\right\rangle$ and pseudorefractive index $\langle N(E)\rangle=\langle n(E)\rangle+i\langle k(E)\rangle$ spectra were measured by spectroscopic ellipsometry from 0.73 to $6.45 \mathrm{eV}$ at room temperature for the light polarization perpendicular to the optic axis $(\vec{E} \perp \hat{c})$. The $\langle\varepsilon\rangle$ spectrum exhibited several interband-transition critical-point structures. Analysis of second-energy derivatives calculated numerically from the measured data yielded the critical-point energy values. (C) 2009 American Institute of Physics. [doi:10.1063/1.3211967]
\end{abstract}

\section{INTRODUCTION}

Gallium selenide (GaSe) crystallizes in a laminar structure characterized by a strong covalent interaction within the layers and a weak van der Waals binding between them as occurs in many other III-VI compounds. The GaSe-type structure can be described as a stacking of hexagonal layers and there are four possible stacking arrangements leading to the four polytypes: $\beta-, \varepsilon-, \gamma$-, and $\delta$-GaSe. ${ }^{1}$ With such a unique structural anisotropy, GaSe has long been an interesting material for both experimental ${ }^{1-12}$ and theoretical ${ }^{1,13-15}$ physics studies, and GaSe and related III-VI compounds have received considerable attention for their potential applications in various device structures. ${ }^{16-20}$ Furthermore, advances in van der Waals epitax ${ }^{21}$ have made it possible to grow single-phase high-quality III-VI compound thin films that renewed interests in GaSe.

Knowledge of optical properties of materials over a wide photon energy range is of importance for designing photonic and photovoltaic devices as well as for verifying the predictions made by the calculations of the electronic energy band structure. Consequently, a number of optical studies of GaSe have been done in the past but with large discrepancies in the results. ${ }^{2-12}$ Early studies ${ }^{2-10}$ reported the dielectric function $\varepsilon$ calculated mostly from the Kramers-Kronig (KK) transformation of the reflectivity data. Spectroscopic ellipsometry (SE) possesses several advantages over conventional reflectivity techniques in terms of the accuracy of data and it has been recognized as a highly suitable method of determining $\varepsilon$ of materials without the use of $\mathrm{KK}$ transformation. ${ }^{22}$ Therefore, SE has been employed widely to investigate optical properties of many semiconductors in the past few decades. $^{23}$

For SE study of GaSe, Meyer et al. ${ }^{11}$ measured both "ordinary" and "extraordinary" $\langle\varepsilon\rangle$ spectra in which the light is polarized perpendicular $(\vec{E} \perp \hat{c})$ and parallel $(\vec{E} \| \hat{c})$, respectively, to the optic axis from 2 to $5 \mathrm{eV}$ under ultrahigh vacuum environment. They observed that the two sets of

\footnotetext{
${ }^{a)}$ Author to whom the correspondence should be addressed. Electronic mail: sukgeun.choi@nrel.gov.
}

optical functions were significantly different and attributed it to the different selection rules for optical transitions. However, the spectral range was limited and no critical-point (CP) analysis was performed. Later, Adachi and Shindo ${ }^{12}$ also reported the ordinary optical response of $\varepsilon$-type GaSe in the similar spectral range $(2-5 \mathrm{eV})$. Although they employed the model dielectric function scheme to analyze their experimental data and extract $\mathrm{CP}$ parameters, only two distinct optical structures at $\sim 3.6$ and $\sim 4.9 \mathrm{eV}$ were studied. More CP structures have been predicted and observed in many other studies. ${ }^{2-10,13,14}$

Here, we applied SE to investigate above-bandgap optical properties of a GaSe single crystal up to $6.45 \mathrm{eV}$ at room temperature. The measured spectrum exhibits several optical structures associated with interband-transition CPs and their energies have been accurately determined by analyzing the numerically calculated second-energy derivative of $\langle\varepsilon\rangle$ within the CP parabolic-band model. ${ }^{24-26}$

\section{EXPERIMENTS}

GaSe single-crystal ( $\varepsilon$-type) used in this study has been grown by the vertical Bridgman method from a melt of stoichiometric GaSe, and then cleaved and cut into a square shape $\left(8 \times 8 \times \sim 0.2 \mathrm{~mm}^{3}\right)$ with the face normal to the [001] direction from the ingot. ${ }^{27}$

The ordinary pseudodielectric function $\langle\varepsilon\rangle=\left\langle\varepsilon_{1}\right\rangle+i\left\langle\varepsilon_{2}\right\rangle$ spectrum was measured from 0.73 to $6.45 \mathrm{eV}$ at room temperature using a spectroscopic rotating compensator-type ellipsometer (M2000-DI model, J.A. Woollam Inc.). The angle of incidence was $70^{\circ}$ and the data were recorded after averaging 1000 compensator cycles (1000 revolutions per measurement) to increase the signal-to-noise ratio.

SE is a surface sensitive technique. ${ }^{22}$ Determination of the material's intrinsic optical properties is therefore complicated by the presence of surface overlayers that generally appear as organic contaminants, a native oxide layer, or microscopic roughness, but it could be any other surface perturbation in practice. Two approaches are usually taken to deal with overlayer artifacts in the experimental spectrum: (1) Mathematical correction within the multilayer model ${ }^{22}$ or 


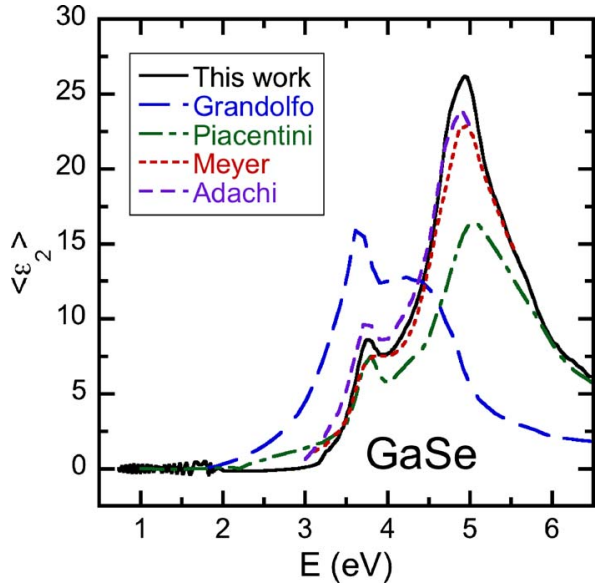

FIG. 1. (Color online) Imaginary part of $\langle\varepsilon\rangle$ spectra for GaSe. The solid line represents the present work. Digitized versions of previously reported data (Refs. 6 and 10-12) are also included for comparison.

(2) actual removal of overlayers using a wet-chemical etching procedure developed by Aspnes. ${ }^{22,28}$ For GaSe, however, its interlayer binding is weak enough to peel the top few layers off easily along the (001) plane, ${ }^{10-12}$ which allows one to obtain a fresh surface immediately prior to SE measurements. The SE data were then acquired under a flowing $\mathrm{N}_{2}$ gas environment to minimize surface contamination during the measurement.

\section{RESULTS AND DISCUSSION}

\section{A. Optical functions}

Figure 1 shows our $\left\langle\varepsilon_{2}\right\rangle$ spectrum for GaSe along with digitized versions of those reported in the literature. ${ }^{6,10-12} \mathrm{~A}$ usual procedure for verifying successful reduction in overlayer artifacts is to maximize the $\left\langle\varepsilon_{2}\right\rangle$ peak in the highenergy region where the penetration depth of the probing light is small and therefore the surface sensitivity is enhanced, ${ }^{28}$ which occurs at $\sim 5 \mathrm{eV}$ for many semiconductors-the $E_{2}$ CP peak. Our $\left\langle\varepsilon_{2}\right\rangle$ value at $4.94 \mathrm{eV}$ is 26.22 which is about $10 \%$ higher than the value reported in the previous SE studies ${ }^{11,12}$ for the same optical structure. Furthermore, a few additional weak optical structures are also seen in the higher energy region $(>4 \mathrm{eV})$ of our data which have not been resolved in the previous SE studies. ${ }^{11,12}$ We thus believe that the spectrum presented here provides a better representation of ordinary $\langle\varepsilon\rangle$ for GaSe than those available in the literature. ${ }^{2-12}$ Our $\langle\varepsilon\rangle$ and $\langle N\rangle$ spectra for GaSe are presented in Figs. 2(a) and 2(b), respectively.

We note that our data show small oscillations in the lowenergy region $(<2 \mathrm{eV})$, which is probably a thickness interference due to the back side reflection from the transparent characteristic of the material below its fundamental absorption edge $\left(E_{g}\right){ }^{29}$ The film thickness estimated from the analysis of the oscillations period ${ }^{30}$ was about $4 \mu \mathrm{m}$, which is much smaller than the actual sample thickness of $\sim 200 \mu \mathrm{m}$. We suspect that a group of layers (with the total thickness of $\sim 4 \mu \mathrm{m}$ ) has partially lost its binding to the remaining bulk due to its weak interlayer binding energy while we peeled off the top few layers. Both the amplitude of

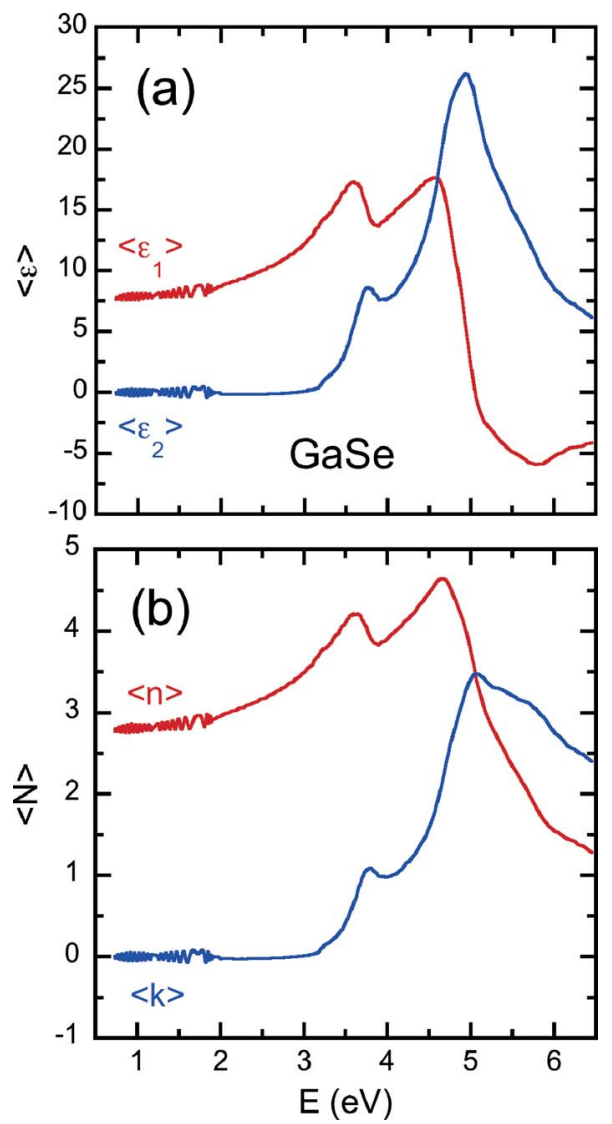

FIG. 2. (Color online) The pseudodielectric function $\langle\varepsilon\rangle$ and the pseudorefractive index $\langle N\rangle$ for $\varepsilon$-phase GaSe.

oscillations and the depolarization (not shown here) are damped to zero at $\sim 2 \mathrm{eV}$, which is indeed a good indication $^{29}$ of the $E_{g}$ of GaSe. ${ }^{1}$

One may expect to see that $\varepsilon_{2}$ and $k$ increase gradually with $E$ for the $E>E_{g}$. As also observed in the previous $\mathrm{SE}$ studies, ${ }^{11,12}$ however, those values for GaSe in Fig. 2 are nearly zero up to $\sim 3.2 \mathrm{eV}$, that is, as much as $1 \mathrm{eV}$ higher than its $E_{g}$. According to theoretical study ${ }^{15}$ of the electronic energy band structure for GaSe, the bottom of the conduction band is formed mainly by the Ga $p_{z}$ state. The top of the valence band is derived from the $\mathrm{Se} 4 p$ states, and the cationcation/anion-anion interactions split this band into the uppermost valence band with $p_{z}$ character and the lower doubly degenerated pairs with $p_{x}, p_{y}$ character, and the spin-orbit interactions further break the degeneracy. The $E_{g}$ of GaSe can then be defined as the interband transition from the uppermost valence band $\left(\mathrm{Se} p_{z}\right)$ to the bottom of the conduction band $\left(\mathrm{Ga} p_{z}\right)$, but the optical selection rules prohibit this transition for $\vec{E} \perp \hat{c}$. A schematic ${ }^{15}$ for the electronic energy band structure at the Brillouin zone (BZ) center in the vicinity of the $E_{g}$ is given in Fig. 3. It is known ${ }^{31}$ that SE measurement of the surface normal to the optic axis carries relatively little information on extraordinary optical property of a uniaxial crystal due to the attenuation of normal component of an applied field by the boundary condition on the displacement vector $\vec{D}$. This is perhaps why SE data do not show a clear optical absorption up to $\sim 3.2 \mathrm{eV}$ where the allowed transition from the deeper valence band $\left(p_{x}, p_{y}\right.$ char- 


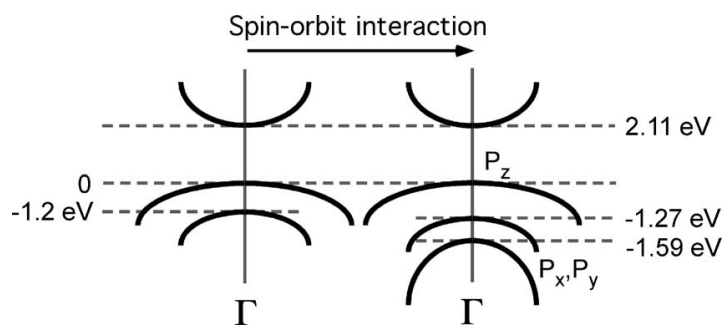

FIG. 3. Schematic of the electronic energy band structure for GaSe in the vicinity of the bandgap $\left(E_{g}\right)$ at the Brilloin zone center (Ref. 15).

acter) to the bottom of the conduction band occurs. Owing to a small contribution from the extraordinary component combining with a quasithin film characteristic in our $\left\langle\varepsilon_{2}\right\rangle$, however, we could make an estimation of the $E_{g}$.

\section{B. CP analysis}

In order to obtain accurate values of the above-bandgap $\mathrm{CP}$ energies, we numerically calculated second-energyderivative spectrum from the measured data using linear filtering algorithms of the Savitzky-Golay type. ${ }^{32,33}$ Here, the data were convolved with a small set of specified weighting coefficients and an appropriate level of smoothing was obtained with 11 data points to suppress the noise level in the derivative spectrum. The $\mathrm{CP}$ energies were extracted by assuming the standard analytic CP expressions for the lineshapes and then fitting them to the data by least squares schemes. These expressions are ${ }^{24,25}$

$$
\frac{d^{2} \varepsilon}{d E^{2}}=\left\{\begin{array}{cc}
n(n-1) A \exp ^{i \Phi}\left(E-E_{g}+i \Gamma\right)^{n-2}, & n \neq 0 \\
A \exp ^{i \Phi}\left(E-E_{g}+i \Gamma\right)^{-2}, & n=0,
\end{array}\right.
$$

where $A$ is the amplitude, $E_{g}$ the $\mathrm{CP}$ energy, $\Gamma$ the broadening parameter, and $\Phi$ the phase angle. The exponent $n$ has the values of $-1,-\frac{1}{2}, 0$, and $+\frac{1}{2}$ for excitonic, one-, two-, and three-dimensional lineshapes, respectively. Detailed discussion on the CP analysis can be found in Ref. 26.

Both real and imaginary parts were fitted simultaneously and the second-energy-derivative spectra of the $\langle\varepsilon\rangle$ together with the best-fit curves are shown in Fig. 4. The open circles and squares represent the $d^{2}\left\langle\varepsilon_{1}\right\rangle / d E^{2}$ and $d^{2}\left\langle\varepsilon_{2}\right\rangle / d E^{2}$ results, respectively, calculated from the measured data, whereas the solid and dash-dotted lines correspond to the best-fit curves of the real and imaginary parts, respectively. Differentiation clearly enhanced the sensitivity to residual features. Seven $\mathrm{CP}$ lineshapes were employed to fit the data ranging from 3 to $6 \mathrm{eV}$, which are indicated by arrows in Fig. 4, and all of those were fitted best with the excitonic lineshapes $(n=-1)$ as evidenced by the lowest mean-square deviations. The $\mathrm{CP}$ energies that we obtained are listed in Table I.

For many semiconductors, assignments of the CP structures are controversial, in particular, when several CP structures are present in a narrow range of the spectrum. ${ }^{34,35}$ The layered structure adds additional complexity for the identification of the origin of each CP structures for GaSe since this material has been described theoretically as either a quasi-two-dimensional $^{36}$ or a typical three dimensional. ${ }^{37}$ Nevertheless, there is a consensus on the origin of the $\mathrm{CP}$ structure at $3.23 \mathrm{eV}$ that corresponds to the transition from

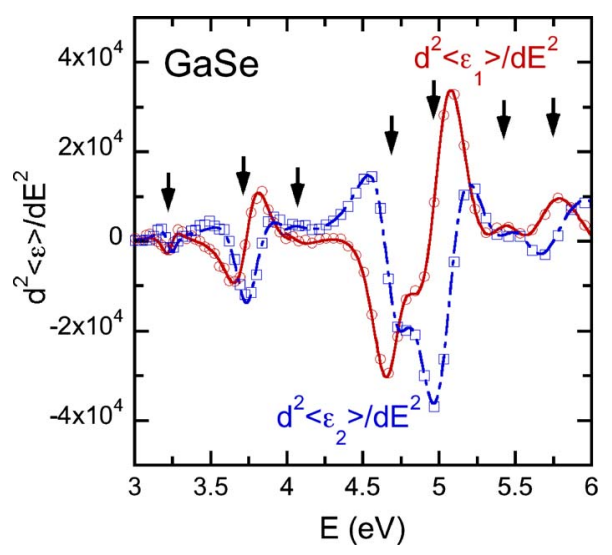

FIG. 4. (Color online) Best-fit curves for the second-energy derivative of $\left\langle\varepsilon_{1}\right\rangle$ (solid line) and $\left\langle\varepsilon_{2}\right\rangle$ (dash-dotted line) of GaSe. The open circles and squares represent data for $d^{2}\left\langle\varepsilon_{1}\right\rangle / d E^{2}$ and $d^{2}\left\langle\varepsilon_{1}\right\rangle / d E^{2}$, respectively. The energy positions of each CPs are indicated by the arrows.

the second highest valence band with the Se $p_{x}, p_{y}$ character to the bottom of the conduction band as discussed in Sec. III A. This assignment implies that the energy separation between this deeper valence band and the uppermost valence band is $1.23 \mathrm{eV}$ assuming the $E_{g}$ is $2 \mathrm{eV}$, which is in an excellent agreement with the theoretical prediction ${ }^{15}$ of $1.27 \mathrm{eV}$ (see Fig. 3).

However, multiple interpretations have been made for the next $\mathrm{CP}$ structure at $3.75 \mathrm{eV}$. It had been once assumed ${ }^{9}$ to be a resonant exciton associated with a two-dimensional $\mathrm{CP}$ involving a transition from the uppermost valence band to the lowest group of the conduction bands along $T$ and $S$ valleys of the BZ, but this assignment appears not to be consistent with the cases for other similar compounds such as InSe. ${ }^{38}$ Piacentini et al. ${ }^{10}$ assigned this CP to the transition from the uppermost valence band to the third group of the conduction band at the BZ center ( $\Gamma$-point). Later, Kuroda et $a l .{ }^{15}$ suggested two deeper valence bands with Se $p_{x}, p_{y}$ symmetry at 1.27 and $1.59 \mathrm{eV}$ below the uppermost valence band with Se $p_{z}$ symmetry for GaSe. The intraband transition of $1.5 \mathrm{eV}$ from the deeper valence band to the uppermost one has recently been observed experimentally by Segura et al. ${ }^{39}$ from their nonlinear optical studies as well as the interband transitions from those two deeper valence bands to the bottom of the conduction band at $\sim 3.3$ and $\sim 3.6 \mathrm{eV}$. Following the results from recent theoretical ${ }^{15}$ and nonlinear optical ${ }^{39}$ studies, we attribute tentatively the origin of the $\mathrm{CP}$ struc-

TABLE I. CP energies for GaSe at room temperature (in eV).

\begin{tabular}{lccccccc}
\hline \hline \multicolumn{1}{c}{ Ref. } & $E_{A}$ & $E_{B}$ & $E_{C}$ & $E_{D}$ & $E_{E}$ & $E_{F}$ & $E_{G}$ \\
\hline This work & 3.23 & 3.75 & 4.03 & 4.69 & 5.02 & 5.45 & 5.72 \\
Ref. 5 & 3.30 & 3.66 & 4.00 & 4.56 & & 5.3 & \\
Ref. 2 & & 3.63 & 4.1 & & 4.9 & & 5.9 \\
Ref. 8 & & 3.67 & 4.25 & & 5.1 & 5.38 & 5.78 \\
Ref. 7 & 3.22 & 3.68 & & 4.78 & 5.07 & 5.48 & 5.75 \\
Ref. 4 & 3.23 & 3.53 & 3.88 & 4.7 & 4.97 & & \\
Ref. 3 & & 3.7 & & 4.8 & & & 5.9 \\
Ref. 6 & & 3.658 & & 4.577 & & & \\
\hline
\end{tabular}


tures at 3.23 and $3.75 \mathrm{eV}$ to the transitions from the two deeper valence bands to the bottom of the conduction band at the BZ center.

The CP structures appearing in the higher energy region have been understood as the excitations of the chalcogen $p_{x}, p_{y}$ electrons to the second and the third conduction bands at the various locations of the $\mathrm{BZ}$ without specific assignments. $^{10,38}$ Positive identification of the origins for those CP structures is beyond the scope of this work. Rather, we intend that this work will help theoreticians perform fine adjustments of the electronic energy band structure of III-VI compounds including GaSe.

\section{CONCLUSION}

Ordinary $(\vec{E} \perp \hat{c})$ optical properties $\langle\varepsilon\rangle$ and $\langle N\rangle$ for $\varepsilon$-phase GaSe single crystal were measured by $\mathrm{SE}$ at room temperature. Good approximations to the intrinsic bulk optical properties were established. Measured spectrum exhibits seven optical structures associated with above-bandgap interband-transition CPs and their energies were obtained by analyzing the lineshapes of the second-energy-derivative spectrum assuming the CP parabolic band model.

\section{ACKNOWLEDGMENTS}

This work was supported by the U.S. Department of Energy under Contract No. DE-AC36-08GO28308. The work done at the Universitat de València was supported in part by the Spanish Project No. MAT2007-66129.

${ }^{1}$ N. C. Fernelius, Prog. Cryst. Growth Charact. Mater. 28, 275 (1994) and references therein.

${ }^{2}$ F. Bassani, D. L. Greenaway, and G. Fischer, Proceedings for the Seventh International Conference on Physics of Semiconductors, 1964 (unpublished), p. 51.

${ }^{3}$ A. Cingolani, C. Manfredotti, A. Minafra, and A. Rizzo, Phys. Status Solidi A 6, 63 (1971).

${ }^{4}$ A. Balzarotti and M. Piacentini, J. Phys. C 4, L273 (1971).

${ }^{5}$ V. V. Sobolev and V. I. Donetskich, Phys. Status Solidi B 45, K15 (1971).

${ }^{6}$ M. Grandolfo, F. Somma, and P. Vecchia, Phys. Rev. B 5, 428 (1972).

${ }^{7}$ S. Kohn, Y. Petroff, and Y. R. Shen, Surf. Sci. 37, 205 (1973).

${ }^{8}$ R. Mamy, L. Martin, G. Leveque, and C. Raisin, Phys. Status Solidi B 62 , 201 (1974).

${ }^{9}$ M. Schlüter, J. Camassel, S. Kohn, J. P. Voitchovsky, Y. R. Shen, and M.
V. Cohen, Phys. Rev. B 13, 3534 (1976).

${ }^{10}$ M. Piacentini, C. G. Olson, A. Balzarotti, R. Girlanda, V. Grasso, and E. Doni, Nuovo Cimento Soc. Ital. Fis., B 54B, 248 (1979).

${ }^{11}$ F. Meyer, E. E. de Kluizenaar, and D. den Engelsen, J. Opt. Soc. Am. 64, 529 (1973).

${ }^{12}$ S. Adachi and Y. Shindo, J. Appl. Phys. 71, 428 (1992).

${ }^{13}$ J. V. McCanny and R. B. Murray, J. Phys. C 10, 1211 (1977).

${ }^{14}$ J. Petalas and J. A. Kalomiros, Phys. Rev. B 44, 8694 (1991).

${ }^{15}$ N. Kuroda and Y. Nishina, Physica B 105, 30 (1981).

${ }^{16}$ G. B. Abdullaev, L. A. Kulevskii, A. M. Prokhorov, A. D. Savel'ev, E. Y. Salaev, and V. V. Smirnov, JETP Lett. 16, 90 (1972).

${ }^{17}$ W. Shi and Y. J. Ding, Appl. Phys. Lett. 84, 1635 (2004).

${ }^{18}$ T. M. Pekarek, C. L. Fuller, J. Garner, B. C. Crooker, I. Miotkowski, and A. K. Ramdas, J. Appl. Phys. 89, 7030 (2001).

${ }^{19}$ M. Afzaal and P. O’Brien, J. Mater. Chem. 16, 1597 (2006).

${ }^{20}$ J. A. Adams, A. A. Bostwick, F. O. Ohuchi, and M. A. Olmstead, Appl. Phys. Lett. 87, 171906 (2005).

${ }^{21}$ J. F. Sánchez-Royo, A. Segura, O. Lang, E. Schaar, C. Pettenkofer, W. Jaegermann, L. Roa, and A. Chevy, J. Appl. Phys. 90, 2818 (2001).

${ }^{22}$ D. E. Aspnes, in Handbook of Optical Constants of Solids, edited by E. D. Palik (Academic, Orlando, 1985).

${ }^{23}$ S. Adachi, Optical Constants of Crystalline and Amorphous Semiconductors: Numerical Data and Graphical Information (Kluwer Academic, Boston, 1999).

${ }^{24}$ M. Cardona, in Modulation Spectroscopy, Solid State Physics, edited by F. Seitz, D. Turnbell, and H. Ehrenreich (Academic, New York, 1969).

${ }^{25}$ D. E. Aspnes, in Handbook on Semiconductors, edited by M. Balkanski (North-Holland, Amsterdam, 1980), Vol. 2.

${ }^{26}$ J. W. Garland, C. Kim, H. Abad, and P. M. Raccah, Phys. Rev. B 41, 7602 (1990).

${ }^{27}$ Ch. Ferrer-Roca, J. Bouvier, A. Segura, M. V. Andrés, and V. Muñoz, J. Appl. Phys. 85, 3780 (1999).

${ }^{28}$ D. E. Aspnes and A. A. Studna, Appl. Phys. Lett. 39, 316 (1981).

${ }^{29}$ M. Garriga, M. Cardona, N. E. Christensen, P. Lautenschlager, T. Isu, and K. Ploog, Phys. Rev. B 36, 3254 (1987).

${ }^{30}$ See, for an example, H. Fujiwara, Spectroscopic Ellipsometry: Principles and Applications (Wiley, New York, 2007), p. 153.

${ }^{31}$ D. E. Aspnes, J. Opt. Soc. Am. 70, 1275 (1980).

${ }^{32}$ A. Savitzky and M. J. E. Golay, Anal. Chem. 36, 1627 (1964).

${ }^{33}$ J. Steiner, Y. Termonia, and J. Deltour, Anal. Chem. 44, 1906 (1972).

${ }^{34}$ S. Logothetidis, M. Alouani, M. Garriga, and M. Cardona, Phys. Rev. B 41, 2959 (1990).

${ }^{35}$ S. G. Choi, C. J. Palmstrøm, Y. D. Kim, D. E. Aspnes, H. J. Kim, and Y.-C. Chang, Appl. Phys. Lett. 91, 041917 (2007).

${ }^{36}$ F. Bassani and G. P. Parravicini, Nuovo Cimento B 50, 95 (1967).

${ }^{37}$ M. Schlüter, Nuovo Cimento Soc. Ital. Fis., B 13B, 313 (1973).

${ }^{38}$ V. Grasso and G. Mondio, in Electronic Structure and Electronic Transitions in Layered Materials, edited by V. Grasso (D. Reidel, Dordrecht, 1986).

${ }^{39}$ A. Segura, J. Bouvier, M. V. Andrés, F. J. Manjón, and V. Muñoz, Phys. Rev. B 56, 4075 (1997). 